\title{
Perspectiva crítica desde latinoamérica: hacia una desobediencia epistémica en terapia ocupacional contemporánea
}

\author{
Juan Pino, Fredy Ulloa \\ Escuela de Terapia Ocupacional, Facultad de Ciencias de la Rehabilitación, Universidad Andrés Bello, \\ Talcahuano, VIII Regiòn, Chile.
}

\begin{abstract}
Resumen: El artículo tiene como objetivo presentar los fundamentos críticos desde los cuales se pueda construir praxis de Terapias Ocupacionales en Chile y Latinoamérica, el manuscrito si bien tiene un desarrollo teórico/conceptual, es el resultado de tensiones que emergen de la práctica profesional cotidiana. Los resultados que se presentan tienen la intención política de invitar a los Terapeutas Ocupacionales a identificar una corriente de conocimiento en común, aplicable en variados campos del actuar disciplinar. Los alcances del trabajo, se deben dimensionar como una pequeña discusión bibliográfica de una corriente mucho más amplia y profunda, que sin embargo, nos entregan varios insumos para la práctica y la teoría e investigación en Terapia Ocupacional. Como conclusión podemos identificar que existe una serie de teorías, metodologías y técnicas que se utilizan en la práctica de la Terapia Ocupacional, pero no se identifican con claridad como una perspectiva desde Latinoamérica, que es lo que viene a proponer el manuscrito e invita a profundizar.
\end{abstract}

Palabras claves: Terapia Ocupacional, Conocimiento, Práctica Profesional.

\section{Perspectiva crítica desde a América Latina: uma desobediência epistêmica na Terapia Ocupacional contemporânea}

Resumo: O artigo tem como objetivo apresentar os fundamentos críticos que embasam a construção da prática da terapia ocupacional no Chile e na América Latina. Embora o manuscrito tenha um desenvolvimento teórico/conceitual, refere-se ao resultado da tensão que emerge da prática profissional cotidiana. Os resultados apresentados têm a intenção política de convidar terapeutas ocupacionais para identificar uma corrente de conhecimento comum, aplicável em vários campos da atuação da disciplina. O alcance do trabalho deve ser dimensionado a partir do limite de sua pequena discussão bibliográfica de uma corrente muito mais ampla e profunda, no entanto nos ofertam várias entradas para a prática e para a investigação teórica em terapia ocupacional. Em conclusão, podemos identificar que há um número de teorias, metodologias e técnicas utilizadas na prática da terapia ocupacional, mas não são claramente identificadas como uma perspectiva a partir da América Latina, que é o que vem propor o presente texto com o convite para aprofundar a discussão.

Palavras-chave: Terapia Ocupacional, Conhecimento, Prática Profissional. 


\title{
Critical perspective from Latin America: an epistemic disobedience in the contemporary Occupational Therapy
}

\begin{abstract}
The article aims to present the critical foundations underlying the Occupational Therapy practice construction in Chile and Latin America. Although the manuscript has a theoretical/conceptual development, it is the result of the tension emerging from daily practice. The results presented have the political intention of inviting occupational therapists to identify a chain of common knowledge, applicable in various fields of the discipline role. The work's scope should be sized as a small bibliographical discussion of a much broader and deeper stream, however, it offers us various inputs for practice and theory and research in Occupational Therapy. In conclusion we can identify a number of theories, methodologies and techniques used in the practice of occupational therapy that are not clearly identified as a particular perspective from Latin America, which is the manuscript proposal, inviting to a deeper discussion .
\end{abstract}

Keywords: Occupational Therapy, Knowledge, Professional Practice.

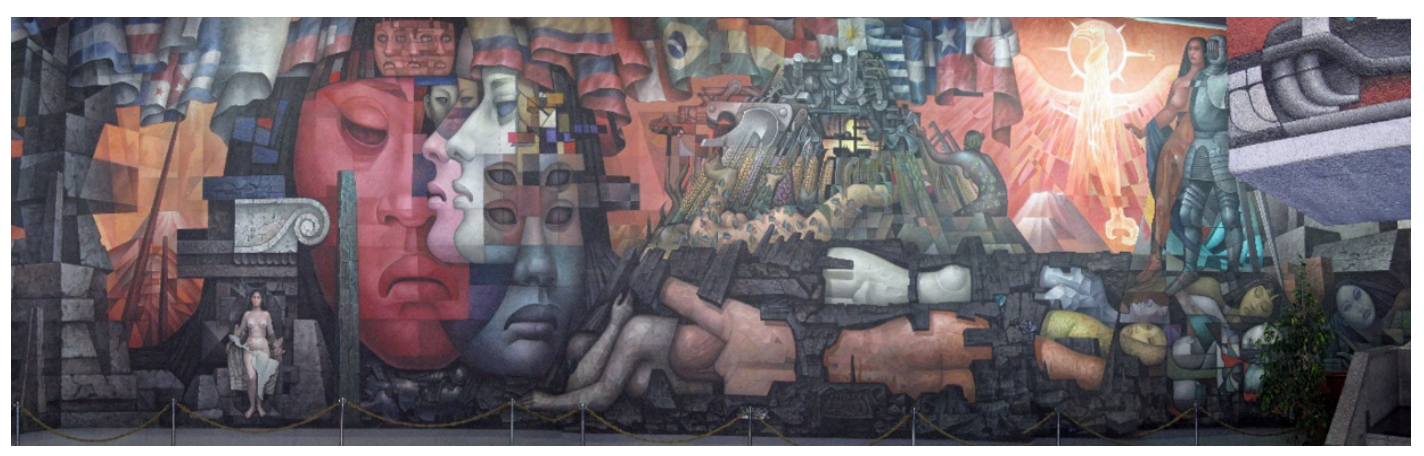

Mural "Presencia de América latina" 1946 Concepcion, Chile.

Soy, Soy lo que dejaron,

Soy toda la sobra de lo que se robaron.

Un pueblo escondido en la cima,

Mi piel es de cuero por eso aguanta cualquier clima.

[...] Soy América latina,

Un pueblo sin piernas pero que camina.

Latinoamérica (CALLE 13, 2011).

La disciplina de la Terapia Ocupacional ha tenido importantes avances en materia de fundamentos filosóficos, epistemológicos y prácticos, con frecuencia en las publicaciones actuales en Sudamérica, especialmente Brasil, Argentina, Colombia, Venezuela, Chile, se encuentran discusiones que interpelan a contar con una posición política, ética, basada en derechos humanos y justicia ocupacional. La emergencia de la Terapia Ocupacional Social, en América Latina, se encuentra en un contexto histórico relevante, posicionándose paulatinamente con mayor fuerza en las prácticas y discursos, la Terapia Ocupacional Comunitaria, por su parte, cada vez cobra mayores espacios, experiencias y sistematizaciones enriquecedoras de su quehacer.
La creciente apertura y formación en el pensamiento crítico de los Terapeutas Ocupacionales, se encuentra en pleno auge y maduración. Por lo tanto, se puede comprender que este conjunto de sucesos y expresiones, responden al ejercicio de una praxis y desarrollo de la Terapia Ocupacional anclada a las necesidades y contextos históricos propios de Latinoamérica.

La variada producción académica en Terapia Ocupacional, ha sido fuente de desarrollo e inspiración para tejer estas nuevas reflexiones y perspectivas, el trabajo y articulación de Sandra Galheigo (Brasil), Alejandro Guajardo (Chile), Salvador Simó (España), Frank Kronenberg (Sudáfrica), Nick Pollard (Inglaterra), Solangel García y Lyda Perez (Colombia), el grupo 
de investigación ocupación y realización humana de la Universidad Nacional de Colombia, entre otros; han influido sustantivamente en develar una perspectiva crítica en Terapia Ocupacional. En este marco, este grupo de Terapeutas Ocupacionales, está incorporando las llamadas "epistemologías del sur", como una nueva forma de conocer y teorizar desde los sures, sin embargo, en el desarrollo teórico de esta emergente perspectiva en Terapia Ocupacional, no queda del todo claro, respecto de cuáles son las fuentes que construyen este pensamiento y perspectiva. El desarrollo del manuscrito pretende ahondar en esa línea, muchas veces difusa y fragmentada; a su vez, queremos colocar en el debate y discusión las influencias esencialmente latinoamericanas, con la idea de comenzar a proponer o denominar una “[...] perspectiva crítica desde Latinoamérica en terapia ocupacional" o como lo destaca la colega Lyda Perez (AGENCIA..., 2014), una terapia ocupacional con enfoque latinoamericano. distinguiéndola de la perspectiva crítica clásica (europea y occidentalista), aún dominante en la mayoría de los trabajos críticos de Terapia Ocupacional.

Para ello trataremos de responder a las preguntas: ¿Se puede pensar en una Terapia Ocupacional crítica desde Latinoamérica?, ¿Cuáles serían sus fundamentos? ¿Quiénes son los autores que trabajan en estas perspectivas?, ¿Cómo nos inspiran para nuestras prácticas y discursos como Terapeutas Ocupacionales?

Este desafío es un incipiente acercamiento desde la Terapia Ocupacional, a una tarea que han realizado diferentes disciplinas y escuelas, un tema abierto al debate, la amplitud de una perspectiva como esta, supera con creces lo desarrollado en este escrito, sin embargo, creemos que puede permitir repensar/afirmar las prácticas y discursos de la Terapia Ocupacional, a su vez, entregar insumos epistemológicos para instalar una perspectiva ausente e invisibilizada en la literatura de la terapia ocupacional actual.

Respondiendo a la primera interrogante, ¿ Se puede pensar en una Terapia Ocupacional critica desde Latinoamérica? creemos que sí, que es posible, y que si es posible, es necesario. De ahí nuestra preocupación por incorporar y construir una perspectiva crítica desde Latinoamérica en las prácticas y discursos de la Terapia Ocupacional contemporánea. Entenderemos la perspectiva crítica desde Latinoamérica como una forma de mirar y analizar la realidad propia de nuestra región y territorio, de una manera situada y global; crítica, en el sentido que quiere reflexionar y problematizar, poner en tensión sus fundamentos hegemónicos, que quiere develar las relaciones de poder en la cuales se ha producido su conocimiento y práctica, a su vez, como estas tecnologías, van generando subjetividades y ocupaciones en los sujetos con los cuales nos vinculamos en este espacio/escenario geopolítico particular; crítica, en el sentido que quiere transformar, modificar las condiciones actuales en las cuales nos desenvolvemos como ciudadanos y terapeutas. Crítica como posición que denuncia, demuestra y rechaza el mantenimiento de condiciones injustas de la vida cotidiana de las personas de esta región. Esperamos que este escrito contribuya al cambio y enriquezca esta respuesta.

¿Quiénes son los autores que trabajan en estas perspectivas? ¿Cuáles serían sus fundamentos? Los fundamentos los podemos encontrar en el llamado "pensamiento filosófico" de nuestra américa latina, desarrollados principalmente a finales del siglo XX e inicio del siglo XXI, con Dussel a la cabeza (1966-2015), como uno de los principales referentes, en conjunto con aportes de varias disciplinas humanistas y sociales, como la Psicología Comunitaria (Maritza Montero), la Psicología de la liberación (Martin Baró), la Sociología Militante y la investigación Acción Participativa (Orlando Fals Borda), la Educación Popular (Paulo Freire), hasta el grupo de estudio modernidad/colonialidad (Walter Mignolio, Aníbal Quijano, Catherine Walsh, Arturo Escobar, Enrique Dussel, Santiago Castro Gomez) como movimiento más contemporáneo, entre otras.

Las ideas que se desarrollan a partir de esta corriente se pueden sintetizar en los siguientes argumentos:

Se considera el "descubrimiento" 2 de América (1492) como un momento histórico determinante para la concepción de la "Modernidad", que trae consigo el "colonialismo/colonialidad" manifestando entonces las primeras versiones del "capitalismo". Galeano (1999, p. 26): se refería

[...] había de todo entre los indigenas de America, pero ninguna de las culturas nativas conocia el hierro ni el arado, ni el vidrio ni la polvora, ni empleaba la rueda; la civilizacion que se abatio sobre estas tierras desde el otro lado del mar vivía la explosión creadora del renacimiento y América aparecía como una invención mas, junto a la imprenta y la brújula daban nacimiento a edad moderna.

Aníbal Quijano (LANDER, 2000, p. 201) señala:

[...] la idea de modernidad, junto con la de evangelización y civilización han sido dispositivos de discriminación, violencia epistémica y en consecuencia, instrumentos de dominación para nuestra región. 
El conjunto de estos procesos sociales dieron curso a la globalización, que en primer término, es la culminación de un proceso que comenzó con la constitución de América y la propagación del capitalismo colonial eurocéntrico, que dieron como resultado un centro hegemónico de poder mundial.

Según Castro Gòmez (2005) era necesario antes que todo construir un discurso sobre el otro y que sin la internalizacion de ese discurso en el habitus tanto de los dominadores como de los dominados, el poder económico y político de Europa sobre sus colonias hubiera resultado inalcanzable.

Siguiendo a Quijano (2000), plantea que uno de los ejes fundamentales de ese patrón de poder es la clasificación social de la población mundial sobre la idea de raza, una construcción mental que expresa la experiencia básica de la dominación colonial. Esto hace percibir como natural y válidas las condiciones injustas de la población de américa, en consecuencia como dados, no susceptibles de ser cuestionados. Esto permea y se aprecia en el conjunto de estrategias eurocéntricas u anglosajonas que se encuentran validadas en la vanguardia de las intervenciones, investigaciones y prácticas de la Terapia Ocupacional actual, al igual como lo describe Lyda Perez (AGENCIA..., 2014) donde

[...] mientras lo individual se refiere a desarrollar un ser autónomo e independiente, la realidad de Latinoamérica exige un estudio de la profesión vinculada a la colectividad, la solidaridad y el trabajo cooperativo.

Said en (CASTRO GÒMEZ, 2005), refiere que la idea de la colonialidad es un elemento constitutivo para comprender la modernidad, representa un punto de vista ideológico sobre la creencia de que la división geopolítica del mundo (centros y periferias) y situa de una perspectiva particular los lugares, los cuales tienen cada uno, privilegios de enunciacion. Asi de un lado está la cultura occidental, presentada como la parte activa, iluminada y donadora de conocimientos, cuya misión es llevar o "difundir" la modernidad por todo el mundo; del otro lado están todas las demás culturas, presentadas como elementos pasivos y receptores de conocimiento, cuya misión es "acoger" el progreso y la civilización que vienen desde Europa, exprexion que se produce en Terapia Ocupacional en la cual sus principales corrientes teoricas provienen del norte y estan en desmedro de los conocimientos del sur, en especial de latinoamericana, vista en el colectivo imaginario como tercermundista, subdesarrollada.

A pesar de lo anterior, en los últimos años se ha instaurado una crítica fructífera a la herencia colonial de occidente, sin embargo, se debe discutir continuamente, en diferentes planos y disciplinas, las premisas de la modernidad en el saber y el hacer, dimensiones centrales de las ocupaciones humanas. El devenir del nuevo orden mundial policéntrico, pensando los nuevos caminos para de-colonizar el pensamiento y las relaciones de poder entre diferentes regiones geoculturales, es una preocupación que debe asumir la Terapia Ocupacional, que en sus expresiones más cercanas, se encuentran incipientes trabajados en interculturalidad, poblaciones inmigrantes, desarrollo local, pobreza y discapacidad, entre otras, quedando aún mucho por hacer en cuanto a la producción de conocimiento desde y hacia las realidades de nuestros pueblos, preocupación elemental para esta posición de la Terapia Ocupacional.

Leopoldo Zea nos advierte que no se trata de seguirle el juego a la retórica modernista, si no instauramos desde lo propio, un pensamiento "sin más”, desde la autenticidad: “[...] en cuanto expresemos nuestro propio modo de ser sin pretender ser otro, colaboraremos en la nunca terminada búsqueda de la libertad y felicidad humana" (ZEA, 1976, p. 223). Es pues una filosofía como conciencia de lo propio, de lo americano "[...] en su expresión histórica y cultural, como en su expresión ontológica" (ZEA, 1991, p. 139).

Por tanto, una de tareas de la Terapia Ocupacional es reivindicar la cultura como una política para el buen vivir, que podemos verla expresada en las ocupaciones y desde nuestras praxis. De esta manera, existirá un compromiso explícito con la

[...] cultura del pueblo, en tanta expresión genuina del potencial de la auténtica liberación. Es en la cultura popular, aún tradicional, que la revolución cultural encontrará su contenido más auténtico (DUSSEL, 1977, p. 147).

En este camino, desde un plano más práctico e interventivo se encuentra materializado el trabajo y aporte del brasileño Paulo Freire (1921-1997), considerado primordial a la hora de investigar en los autores latinoamericanos, su compromiso con los oprimidos, le permitió generar mecanismos de concientización y educación para lograr la transformación de grandes grupos de población brasileña y latinoamericana. La educación para la liberación, las estrategias activo-participativas, los procesos de acción-reflexión, se reconocen como una de los más grandes aportes de la educación popular. Cobran gran importancia dentro de sus múltiples obras La educacion como práctica de la libertad (FREIRE, 2009), con los argumentos de educación versus masificación; educación y 
concientización; y educación práctica (aprender haciendo), y La pedagogía del oprimido (FREIRE, 2012), con explicaciones a la concepción bancaria de la educación como instrumento de opresión; la concepción problematizadora respecto a la idea de que nadie educa a nadie, sino, que los hombres se educan entre sí con la educación del mundo; y la teoría de la acción dialógica y sus características, son algunos de las reflexiones y experiencias que Freire nos entrega.

En el mismo plano, el aporte que nos entrega el colombiano Orlando Fals Borda (1925-2008). El cual destaca en sus obras la conjunción de la indagación sociológica con el compromiso político en beneficio de los "sectores populares". Sus ideas pueden profundizarse en obras como Conocimiento y poder popular (FALS BORDA, 1985), mostrando en ella aportes en torno al saber interactuar y organizarse; rompiendo la relación de sumisión; valoración y empleo de la cultura popular; develando nuevas experiencia en la investigación y la acción participativa, ejemplificando el caso de la localidad el Cerrito, en Colombia. Así mismo, ideas en como investigar la realidad para transformarla; la crisis, el compromiso y la ciencia; y experiencias teórico prácticas, encontradas en su obra Una sociología sentipensante para américa latina (FALS BORDA, 2009), son ejemplos de su producción intelectual comprometida. Sin duda su obra reciente Globalización y segunda república (FALS BORDA, 2008b), aporta claras ideas para comprender las vertientes de la globalización, apelando a estrategias como la globalización como respuesta para combatir el eurocentrismo, tan vivo en nuestra actualidad.

Siguiendo la misma tradición se encuentra el trabajo de Martin Baró (1942-1989) en el Salvador, el cual luchó por los derechos humanos, y la justicia social. Fue un seguidor de la teología de la liberación y padre de la psicología de la liberación, referente de la psicología social latinoamericana. Defendió la tesis de que la psicología debería establecer una estrecha relación con las condiciones sociales e históricas del territorio donde se desarrolla, y asimismo, con las aspiraciones de las personas que en él residen. Dentro de sus obras se destaca Psicodiagnóstico de América Latina (BARÓ, 1972), publicado por la Editorial de la Universidad Centroamericana en 1972. Los dos tomos de Acción e Ideología (BARÓ, 1983, 1989). Donde recalcó el rol político y el compromiso para enfrentar las condiciones injustas de la población salvadoreña, destacó su profundo sentido de vida para revindicar a los oprimidos de Centroamérica, su trabajo de concienciación y desenmascaramiento fue una constate en su legado social.
En la actualidad y bajo la influencias del trabajo de los anteriores, se destaca el trabajo de la venezolana Maritza Montero, la cual es una de las figuras pioneras de la psicología comunitaria latinoamericana. Sus producciones consisten en originales desarrollos en Psicología Social Comunitaria, Psicología Política y Crítica, y Psicología de la Liberación, los que se caracterizan además por la riqueza de sus investigaciones y análisis, donde primordialmente se devela y destaca el rol de la comunidad como agente de transformación y cambio social. Teoría y práctica de la psicología comunitaria la tensión entre comunidad y sociedad (MONTERO, 2003) resaltando las lógicas del poder en la intervención comunitaria, los tipos de liderazgo, el carácter político del trabajo psicosocial comunitario y las redes comunitarias, Hacer para Transformar (MONTERO, 2006), el foco específicamente en el aspecto metodológico de la intervención, las técnicas más frecuentes en el trabajo comunitario (entrevistas participativas, discusiones reflexivas, anotaciones de campo, documentos audiovisuales), en conjunto con el proceso de familiarización con la comunidad, la jerarquización de las necesidades y los recursos, como herramientas para la praxis.

\section{¿Cómo nos inspiran para nuestras prácticas $\mathrm{y}$ discursos como Terapeutas Ocupacionales?}

Reflexionando lo anteriormente revisado y parafraseando a Mignolo (2010, p. 210), es urgente adoptar una "desobediencia epistémica en Terapia Ocupacional contemporánea”, lo cual implicaría tomar una posición ética-política-cultural radical, en sentido que busque identificar y solucionar las problemáticas desde su raíz, es un desprendimiento epistémico en la esfera de lo social, es un movimiento que sale de la comprensión universalista hegemónica, hacia una pluriversatilidad de la Terapia Ocupacional.

Esto nos obliga a posicionarnos desde Latinoamérica, que como plantea el Manifiesto Latinoamericano de Terapia Ocupacional y Ocupación (RAMÍREZ; SCHLIEBENER, 2014), no pretende en ningún caso asumir una posición chovinista, auto excluyente, antojadizo ni confrontacional con las Terapias Ocupacionales del resto del mundo. Al contrario se plantea que es la única manera de poder favorecer procesos de integración y solidaridad a nivel mundial, es reconociendo y comprendiendo que nuestra historia y nuestra realidad no es la misma que en Suecia, Finlandia, Canadá, Australia ni EEUU. 
Lo anterior, quedo claramente plasmado en el Congreso Mundial de Terapia Ocupacional, realizado en Chile el 2010, donde Galheigo y Simó (2012) sostuvo, que provocó una repercusión importante en la TO internacional, este evento promovió un cambio importante en lo que se reconocía sobre la perspectiva anglosajona era legítima mundialmente. No cabe duda que la importancia del congreso fue la de mostrar que la Terapia Ocupacional, ha sido desarrollada desde décadas en los países del sur, comprendiendo y ejerciendo su acción en consonancia con su realidad social. Creemos al igual que Galheigo y Simó (2012), que son las acciones profesionales las que deben ser pensadas para atender a las necesidades y demandas de la población, y ser desarrolladas en un proceso participativo, democrático, dialógico, como se plantea desde la educación popular que propone Freire (2012). Se considera que una profesión constituye sus saberes y prácticas en respuesta a las necesidades de las personas y colectivos, y éstas necesidades de los países del sur se muestran diferentes a las de los países del norte. Sumándole que cuando hay determinantes estructurales fuertes, una TO centrada en el cliente acaba por desempoderar a las personas y sumiéndolas en un vacío ocupacional.

Ramírez y Schliebener (2014), nos llaman a posicionarnos desde el sur y proponer una Terapia Ocupacional diferente, rescatando nuestra memoria histórica como pueblo y reconocernos en los mejores aspectos de este, en la lucha, la solidaridad, las alegrías, los sueños, utopías y las riquezas que nos han permitido resistir siglos de colonialismo, dominio, opresión y explotación; a recuperar nuestros discursos propios, a contar nuestra propia historia, leyendas, alzando nuestros propios mitos e inventando nuestro propio futuro y conocimiento, en palabras de Santos (2009) son globalismos localizados.

En vista de ello, se requiere desarrollar una Terapia Ocupacional de la Liberación, la cual no es un pasatiempo de los "nuevos intelectuales" como se refiere Pollard (2013), bajo esta perspectiva no se busca una demostración de habilidades retóricas sobre vanas especulaciones de un pensador que vive encerrado en su equipo profesional. El Terapeuta Ocupacional aquí, ni siquiera es un hombre que piense porque ama la sabiduría; el terapia ocupacional de la liberación se compromete como lo hizo Martín Baró, con el hombre sufriente, con los pueblos explotados y enajenados de sus derechos y ocupaciones, y piensa el porqué de su condena, así como la estrategia para superar esta injusticia, pues lleva en su interior siempre la intención de la transformación.

Desde ahí, la "liberación" es un término geopolítico clave en el marco de los movimientos sociales de "liberación nacional" en África, Asia y América
Latina, incluye dos tipos de proyectos diferentes e interrelacionados: la descolonización política y económica, y la descolonización epistemológica; a diferencia de "emancipación" que pertenece a la Ilustración europea y su noción es compartida por los discursos tanto marxistas como liberales y aún se sigue usando en la misma tradición Mignolo (2010).

Desde esta comprensión de la terapia ocupacional, se propone "descolonizar la ocupación humana" que es el desplazamiento, la noción de desprendimiento hacia la condición pluriversatil de la ocupación; comprender la ocupación como expresión histórica diversa, como praxis en constante transformación, como potencial humano que va mutando a través de las tensiones y luchas sociales, a través de los cambios de paradigmas y el desarrollo de las fuerzas productivas. Estas transformaciones se generan al interior de comunidades, que como plantea Montero, es el carácter político del trabajo psicosocial comunitario y la activación de las redes comunitarias que gatillan ocupaciones descolonizadas, y que a su vez, producen significados de las ocupaciones con sentido colectivo, condición sine qua non en propuestas latinoamericanas de TO.

Una ocupación humana con calidad localista del conocimiento, que abre un portillo para combatir los malos efectos del eurocentrismo, determinar sus flancos débiles y enfrentarlos con fuerzas territoriales de resistencia. Localismos globalizados en contra de globalismos localizados del conocimiento para la Terapia Ocupacional, convergen hacia la glocalización de la ocupación y la investigación. Esta metodología, que propone Fals Borda (2008a) de investigación acción participación, compromete al investigador con la acción, donde la participación con las personas está dada por la necesidad de transformación, la preocupación principal no se genera por un interés intelectual y académico, sino, más bien por la necesidad de aprender a resolver las problemáticas de la vida cotidiana. Esta respuesta es ventajosa y diferencial del origen por nuestro saber local, genética e historia, como arma estratégica y de rebeldía para descolonizar la Ocupación Humana.

La descolonización de la ocupación, nos interpela como Terapeutas Ocupacionales, ya que, somos nosotros/nosotras los actores involucrados quienes tienen en común la experiencia de la herida colonial,

[...] la experiencia de las y los no adecuados al orden 'normal' de la sociedad. Hoy más que nunca necesitamos "aprender a desaprender para poder así re-aprender (MIGNOLO, 2010, p. 98).

Para finalizar, queremos agradecer al grupo de investigación vida cotidiana, que nos permitió tener los espacios para reflexionar y problematizar sobre los posibles fundamentos de la TO desde 
Latinoamérica. Este ejercicio preliminar logró ser plasmado con la intención de invitar a construir mayores insumos teóricos prácticos para nuestras praxis como trabajadores y actores sociales.

\section{Referencias}

AGENCIA DE NOTICIAS UN. Terapia Ocupacional requiere enfoque latinoamericano. Concepcion: Universidad Nacional de Colombia, 2014. Disponible: <http:// agenciadenoticias.unal.edu.co/detalle/article/terapiaocupacional-requiere-enfoque-latinoamericano.html>. Acceso: 14 dic. 2015.

BARÓ, M. Psicodiagnóstico de América Latina. San Salvador: UCA Editores, 1972.

BARÓ, M. Acción e ideología: psicología social desde Centroamérica. San Salvador: UCA Editores, 1983.

BARÓ, M. Sistema, grupo y poder: psicología social desde Centroamérica II. San Salvador: UCA Editores, 1989.

CALLE 13. Latinoamérica. Intérprete: Calle 13. In: CALLE 13. Entren los que quieran. [s/l]: Sony BMG, 2011. Faixa 7.

CASTRO GÒMEZ, S. La Hybris del Punto Cero. Bogotá: Editorial Pontificia Universidad Javeriana, 2005.

DUSSEL, E. Para una ética de la liberación latinoamericana. Buenos Aires: Siglo XXI, 1977.

FALS BORDA, O. Conocimiento y poder popular. Bogotá: Siglo XXI ediciones, 1985.

FALS BORDA, O. El socialismo raizal y la gran Colombia bolivariana. Caracas: Editorial El Perro y la Rana, 2008a.

FALS BORDA, O. Globalización y segunda república. Buenos Aires: CLACSO, 2008b.

FALS BORDA, O. Una sociología sentipensante para américa latina. Bogotá: Siglo del Hombre Editores, 2009.

FREIRE, P. La educación como práctica de la libertad. Argentina: Siglo XXI Ediciones, 2009.
FREIRE, P. La pedagogía del oprimido. Rio de Janeiro: Siglo XXI Ediciones, 2012.

GALEANO, E. Las venas abiertas de américa latina. Montevideo: Siglo XXI Ediciones, 1999.

GALHEIGO, S.; SIMÓ, S. Maestras de la terapia ocupacional. Sandra Galheigo: la poderosa emergencia de la terapia ocupacional social. Revista TOG, Galícia, v. 9, n. 15, p. 1-41, 2012.

LANDER, E. La colonialidad del saber: eurocentrismo y ciencias sociales: perspectivas latinoamericanas. Buenos Aires: CLACSO, 2000.

MIGNOLO, W Desobediencia epistémica: retórica de la modernidad, lógica de la colonialidad y gramática de la descolonialidad. Alpha, Osorno, n. 35, p. 210-214, 2010.

MONTERO, M. Teoría y práctica de la psicología comunitaria: la tensión entre comunidad y sociedad. Buenos Aires: Editorial Paidós, 2003.

MONTERO, M. Hacer para transformar: el método de la psicología comunitaria. Buenos Aires: Editorial Paidós, 2006.

POLLARD, N. Escritura y narrativa: una comprensión hacia las ocupaciones cotidianas. In: CONFERENCIA DIA DEL TERAPEUTA OCUPACIONAL, 2013, Concepción. [s.l.]: [s.n.], 2013. Presentación oral.

QUIJANO, A. Colonialidad del poder, eurocentrismo y América Latina: perspectivas latinoamericanas. Buenos Aires: CLACSO, 2000.

RAMÍREZ, R.; SCHLIEBENER, M. Manifiesto latinoamericano de terapia ocupacional y ocupación. $R e-$ vista TOG, Galícia, v. 11, n. 19, p. 1-18, 2014.

SANTOS, B. S. Una epistemología del Sur: la reinvención del conocimiento y la emancipación social. México: Siglo XXI, 2009.

ZEA, L. Filosofia y cultura latinoamericanas. Caracas: Centro de Estudios Latinoamericanos "Rómulo Gallegos", 1976.

ZEA, L. La filosofia como compromiso de liberación. Caracas: Biblioteca Ayacucho, 1991.

\section{Contribución de los Autores}

Juan Pino Morán y Fredy Ulloa participarán en la concepción y diseño del trabajo, recolección y obtención de los datos, análisis y discussion de los resulados, redacción del manuscrito y revision crítica de éste. Todos los autores aproaban la version final del texto.

\section{Notas}

${ }^{1}$ Se puede revisar con mayor detalle en el trabajo de Boaventura de Sousa Santos (2009), en Una epistemología del Sur. La reinvención del conocimiento y la emancipación social.

${ }^{2}$ Idea que es desarrollado bajo una cosmovisión europea, transmitida por la historia tradicional occidentalista, contraria a la idea de colonialismo, invasión, saqueo y exterminio de la cultura indígena. 